\title{
Information Spaces for Mobile City Access
}

\author{
Philip Berridge ${ }^{[a]}$, Volker Koch ${ }^{[b]}$, and Andre G. P. Brown ${ }^{[a]}$ \\ [a] CAAD Research Unit, \\ School of Architecture and Building Engineering, \\ The University of Liverpool, UK \\ p.berridge@liv.ac.uk \\ [b] Institut für Industrielle Bauproduktion, \\ Fakultät für Architektur, \\ Universität Karlsruhe (TH), Germany \\ volker.koch@ifib.uni-karlsruhe.de
}

\begin{abstract}
This paper describes two complementary European research projects that share common interests and goals. The work described is intended to facilitate city analysis and support decision-making. The first project focuses on the distribution, access and ease of use of city data. The system allows access to historical data concerning key buildings in Liverpool, England, via a suite of web-based tools and a palmtop device. The second project looks at extending the functionality of traditional computer aided design (CAD) software to enable geometric and semantic data to be combined within a single environment. The system allows those involved in city planning to better understand the past and present development pattern of an area so that their decisions on future proposals are better informed. The paper concludes by describing a system that integrates particular aspects of the two projects, and the potential that this integration can bring. This new work provides mobile access to historical city development data, current city information and tools to support urban project development.
\end{abstract}

\section{INTRODUCTION}

Cities and fragments of cities are constantly evolving. These changes usually happen slowly as cities and their management structures respond to changes in economic, political, social, cultural and environmental conditions. Occasionally, major events bring radical shifts, producing unexpected changes to the cityscape.

Cities can only be properly understood within their historical context [1]. However, localised planning and development strategies, that seek to impose a structure on the evolutionary process, may fail to take into account the existing urban structure and its history. The development of the Internet has assisted with the dissemination of historical and other forms of city data but the information available is often patchy and unstructured. In addition, as the urban development process is opened up to a wider group of participants with different specialisations, a holistic discussion of the issues becomes more difficult as there is often little shared understanding of all the factors that must be considered, between all the participants involved $[2,3]$. Creating a commonly understood 
framework is a key to improving discussion and understanding amongst groups from a disparate range of backgrounds.

The complementary projects described in this paper aim to increase the understanding of cities by linking often complex semantic data with an easily understood visual representation. The projects use three-dimensional models of the cityscape as information spaces that enable the visualisation of elements of both the historic and contemporary aspects of economic, political, social, cultural and environmental issues. This visualisation of complex data concerning the past and present state of a city or area facilitates a shared framework of understanding among both experts and non-experts involved, for instance, in the planning process. In turn, this enhancement of understanding aids analysis and supports urban planning bodies in making appropriate decisions concerning the future development of a city.

Although a key focus of the projects is their potential impact on the planning process the work demonstrates other useful applications of information spaces as tools to present information and aid understanding.

\section{ARCHITECTURAL HERITAGE: LIVERPOOL}

This project aims to integrate available strands of city data in order to improve both access and the transfer of high-level information. It uses a pseudo three-dimensional model of the City of Liverpool as an information space allowing a database of city information to be navigated using an easily understood visual representation. The techniques used can be applied to many types of city data; the prototype referred to in this paper uses data concerning the city's architectural history.

\subsection{Context}

Liverpool is a city with a rich architectural heritage stemming from its Maritime past $[4,5]$. It has more grade one listed buildings than any other UK city outside London. A thousand of its best buildings, centred on the waterfront and cultural quarter, form the UK government's only current nomination for World Heritage Site status submitted to the United Nations charity, UNESCO. This area is the focus of the prototype.

Many cities have implemented digital city information spaces delivered via the Internet on desk-based or mobile devices. The majority of desk-based systems, such as Virtual Glasgow [6, 7, 8] and Virtual Cheltenham [9], use VRML or other specialised web plugins to allow access to the 3D model and city data.

More recently there has been experimentation and development of mobile city guides with 3D capability such as those by Belz et al. [10] and Raposo et al. [11] and the Deepmap project [12]. Rakkolainen et al. [13] have developed their system to visualize real-time Global Positioning System (GPS) data through VRML worlds. The LoL@ project aims to implement a location-based electronic multimedia tourist guide for Vienna based on UMTS (Universal Mobile Telecommunications Systems) and GPS [14]. The LoL@ uses a 2D city map as a visual location guide and a key objective of this project was the improvement (speed and quality) of data access. Although the specialised web plug-ins or hardware used by most of the 3D-based information spaces can increase their functionality, their accessibility is reduced, especially to users of less common operating systems. This work parallels and augments existing systems by integrating 
associated digital technologies that have been widely adopted both inside and outside urban planning disciplines.

The Liverpool project, described in this paper, has been implemented by integrating two complementary environments; a desk-based system and mobile PDA-based system. At the heart of both implementations is an existing database of information on key historical buildings. This was compiled during a previous strand of the research [15]. Both the desk-based and PDA-based systems use the pseudo three-dimensional model to improve the usability of, and access to, the database.

\subsection{The desk-based city navigator}

Rakkolainen et al. [13] have shown that three-dimensional models enable users to understand cityscapes and recognise locations better than symbolic 2D maps. The objectives of the system described in this paper are to enable users to interrogate a database using a three-dimensional model and to provide users with a visualisation of database query results. Within the model landmark and historically significant buildings are represented to provide key visual cues when wayfinding.

The system runs within a web browser using standard HTML and Macromedia Flash technology. It uses a small window to display an easily understood pseudo 3D model of the city. Only a portion of the model can be viewed on screen at any one time but users can freely navigate around, selecting buildings to access their database query result. In the prototype, when a user moves the cursor over a building basic data relating to that building is displayed. When a user clicks on a building the full database entry is displayed. The 3D model makes the background database much easier to browse. Access is improved and users can relate the data within the context of the city's urban framework.

In addition to using a three-dimensional model as a database browser, the system allows the data to be queried using a standard html-based form. In this scenario the query results are presented in text form and, more importantly, within the 3D environment. In the prototype, users can search the database for known buildings or buildings that possess certain qualities. For example, a user can select a building they wish to locate using a HTML form and the system will visualise the query results by automatically navigating until the required building and its immediate environment in the model are visible. Supplementary text and images from the database are also displayed. Consequently, the three-dimensional computer visualisation supports real-world navigation by making points of user interest easier to locate.

\subsection{The PDA-based city navigator}

Part of the project has been the customisation of the city navigator for mobile users. This work aims to exploit existing handheld computing technology and has centred on allowing access via a palmtop device so that users can access the building data on location. Much of our recent research has concentrated on using readily available, lowcost and generic technology to provide access to virtual worlds and 3D models at a citywide and building specific level [16]. This current research applies the same strategy so as to make the data accessible to the widest range of potential users. The prototype uses AvantGo for data viewing as it is installed as standard on many palmtop devices. 
An initial palm-based system developed during a previous strand of the research [15] facilitates on-site access to walking tours of the city's key buildings [17]. The system uses a three-dimensional representation of the city to supplement text-based information extracted from a database. The PDA city navigator prototype builds on this earlier work. It provides a 3D representation of the cityscape, which the user can freely navigate by clicking and dragging (Figure 1). Buildings with database entries are shown within the context of the city's urban fabric making them easier to locate.

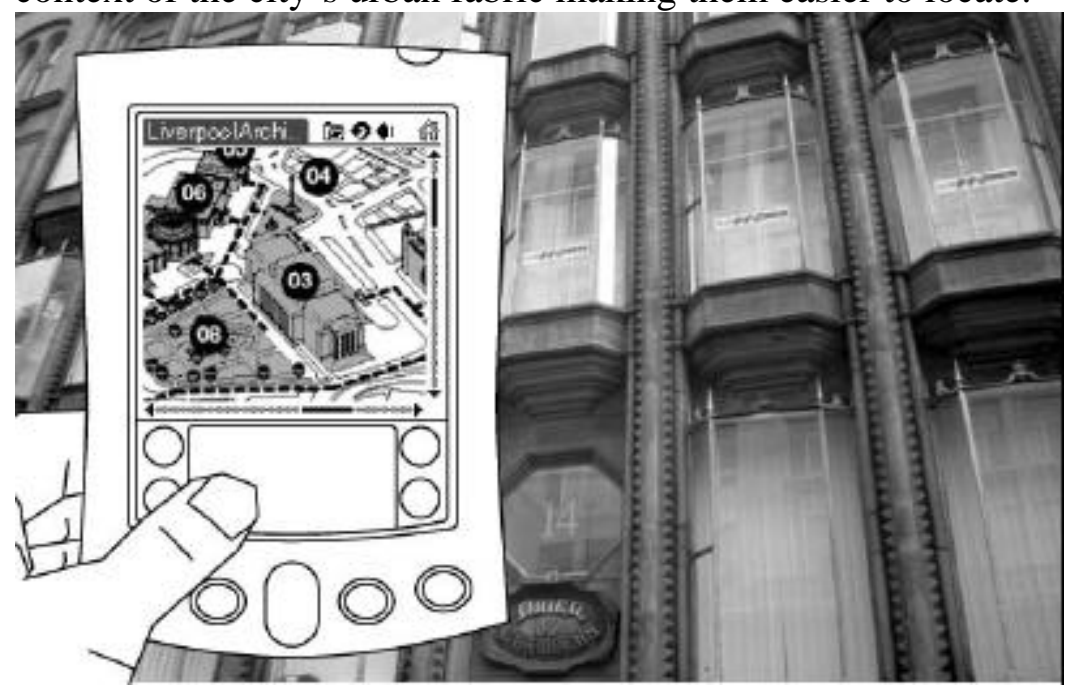

Figure 1: The PDA-based city navigator showing the city's museum quarter. Oriel Chambers, designed by Peter Ellis in 1864, and one of the many significant buildings in Liverpool, is shown in the background.

In addition to allowing users to see the context of each building and the routes between them, the PDA city-navigator, like the desk-based system, facilitates direct access to individual building data. The user simply clicks on (or points at) a building to access its database entry.

\subsection{Access philosophy}

Over recent years, as wireless communication and palmtop technologies have improved, delivering wireless content has become a viable option. The more recent mass production of integrated mobile telecommunication and palmtop devices has also served to increase the potential number of wireless content users.

Despite these continuing developments, currently our prototype does not allow remote wireless access to the architectural history database. Instead in order to maximise the potential user base, a core objective of the project, the system works in 'disconnected' mode; the user must choose the data they wish to install when they synchronise their device via an Internet connected desktop PC. This creates a stand-alone portable system on the PDA that has performance benefits. In addition it can be accessed by the large subset of palmtop users who have legacy devices with no means of wireless communication or who simply do not use their device's wireless capabilities. Consequently, the current system is limited as it does not allow dynamic interaction with the database on location [18]. 
However, the conversion of the system from disconnected to fully wireless ('connected') is straightforward to implement within the AvantGo environment. But, as the transfer of city data could be accessed through transfer channels of differing capacity, a hybrid system may still be more appropriate in which some data is sent to mobile devices on demand and a subset installed directly on the mobile device. We envisage the system described here being developed in this way.

Currently, when a user installs the system a default data set is downloaded to their device. This consists of the 3D city-navigator and the data for each building on one of the walking tours. However, users can customise the building data they install on their palmtop using customisation options [19]. Both single and groups of buildings can be added or removed (Figure 2). Users are warned if removing a building will make either of the walking tours incomplete. In addition to the main customisation options, single buildings can be added via the city navigator. When a user clicks on a building for which data is not installed a simple form is displayed allowing the user to add that building to their set-up.

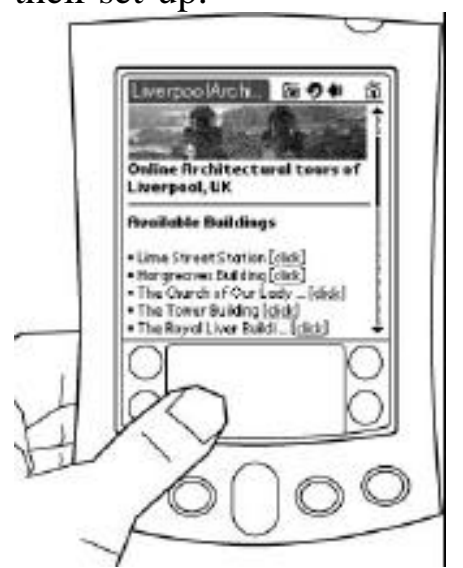

Figure 2: The PDA-based system showing the buildings currently installed.

As the prototype has been developed as a disconnected system, when one of the off-line user customisation forms is submitted it is not sent directly to the web server. The prototype uses the AvantGo 'forms manager' to store the form submissions, which sends the data contained in the forms the next time the user synchronises their device (i.e. goes on-line and connects the PDA to the desktop system). For authorised editors, during synchronisation the new content is downloaded and updates to previously installed records are retrieved.

\subsection{Multiple information sources}

The systems described both use a single Internet distributed database of architectural data. However, city data, even concerning a single subject matter, is rarely contained within a single source. Our prototypes both use CGI (Common Gateway Interface) scripts to interface the three-dimensional model with the dataset. The systems also integrate well with other standard web-based database query methods such as those using SQL, PHP and XML [20].

We are currently working with a number of local and regional organisations to adapt the techniques in order to provide access to other types of city and building data. This work 
will use the core three-dimensional model as a basis for integrating many strands of available city data further improving information access.

\section{TIME-SENSITIVE PLANNING: KARLSRUHE}

The second project seeks to provide both city planners and students with a data capture and analysis tool to support the reference to the past, analysis of the present and development in the future, of urban elements and issues. It enables planning teams to view the urban objects of study over a period of time beginning with their construction. The tool is accessed within an existing and widely used CAD environment (AutoCAD) and uses a three-dimensional model of a city fragment as the reference vehicle. The basis of the implemented tool is the linkage of geometrical objects (modelled buildings) with semantic information within a CAD environment. In the prototype, data concerning the building's lifespan is stored, such as the date of construction, information regarding any additions to the building and demolition date. However, any supplementary information that can be gathered about a building and its immediate context can be incorporated (Figure 3). All data is organized within a database that is linked with the geometrical object to which it relates.

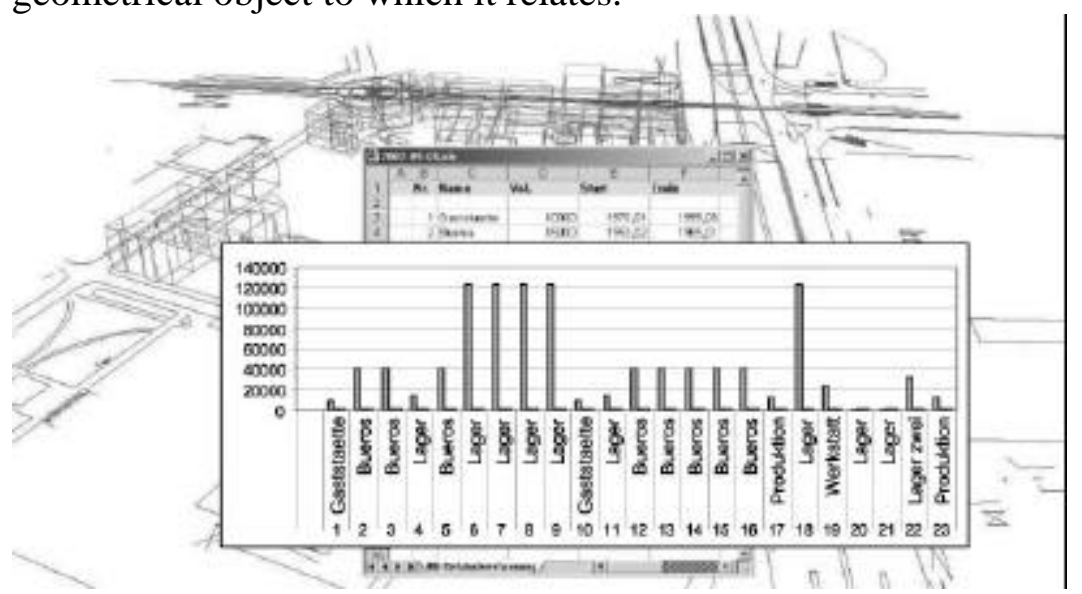

Figure 3: Connecting geometrical objects with time information.

The linking of the geometric and the semantic data allows a modelled city fragment to be visualised over time. This allows the analysis of time-lines of development and change and creates a user-friendly working information space that allows experts and nonexperts to have a better-informed and productive dialogue.

\subsection{Phases of work}

The system's workflow can be split into four stages; modelling, data input, visualisation and analysis. In the first phase urban models are created within standard CAD applications. Each building, or other urban form, is represented by a single CAD object or group of objects. These models can be created in two or three-dimensions. However, as discussed above, three-dimensional models increase understandability, particularly for non-planning professionals. The prototype uses a three-dimensional model.

Phase two requires the entry of related data. The modelled geometrical objects are linked to semantic information, including building history, spatial modifications, usage and 
condition. Additional photographic, video and other information can also be attached to the objects in the model database.

The visualisation phase allows the development of the urban fabric to be viewed over time. A snapshot of the modelled environment at a user-specified single point in history can be viewed. Alternatively, an animation showing the change to the chosen city fragment over time can be created. For the latter, static snapshots at the start and end date of the required view period are selected along with the number of in-between steps required. In both cases the visualisation of the urban fabric within the CAD environment can be modified many times via additional parameters, such as the one describing the condition of the buildings.

The different ways of visualising the geometrical and semantic information allow the planning team a multiplicity of representations of past, existing or planned development. This greatly enhances discussion and analysis of planning policy. In short, the visual representation of an urban area over a time period that is supported by an extensive database provides an extremely useful support tool for designers, planners and students in these areas.

\section{INTEGRATING THE TWO PROJECTS}

The current goal of the research is the integration of the two desk-based systems with the PDA-based system to create a mobile time-sensitive device. The new project aims to develop and implement a mobile interactive system using a three-dimensional information space as a way to access data on the information describing an area whilst on location.

A substantial feature of the work is the integration of historical and planned (future) urban development with information on the current situation. This will show users the ongoing evolution of the urban fabric allowing both citizens and planners to evaluate future proposals within their historic context (Figure 4).

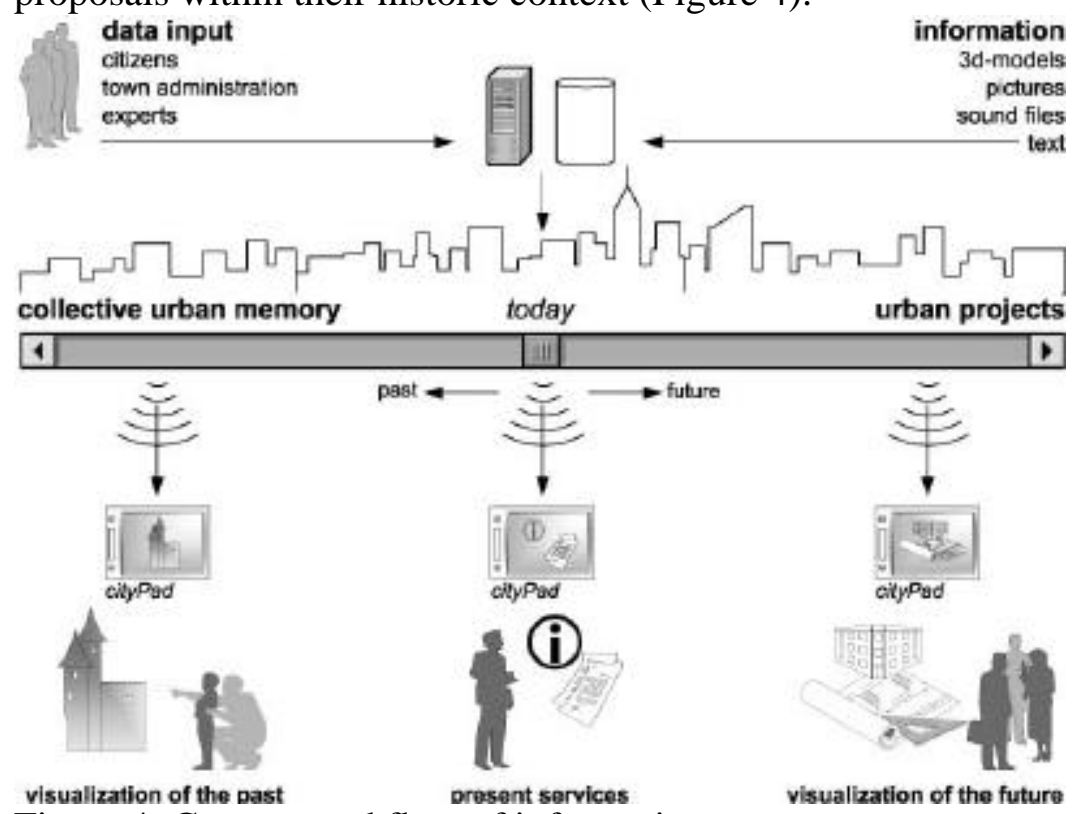

Figure 4: Concept and flow of information. 
Access to the urban information spaces will take place via modified and extended handheld devices (cityPADs) with geographical positioning capabilities. Whilst the user is on-site in the real urban environment the device will provide access to a mix of associated information allowing them to inspect location dependent data that is usually not readily available on-site (Figure 5).

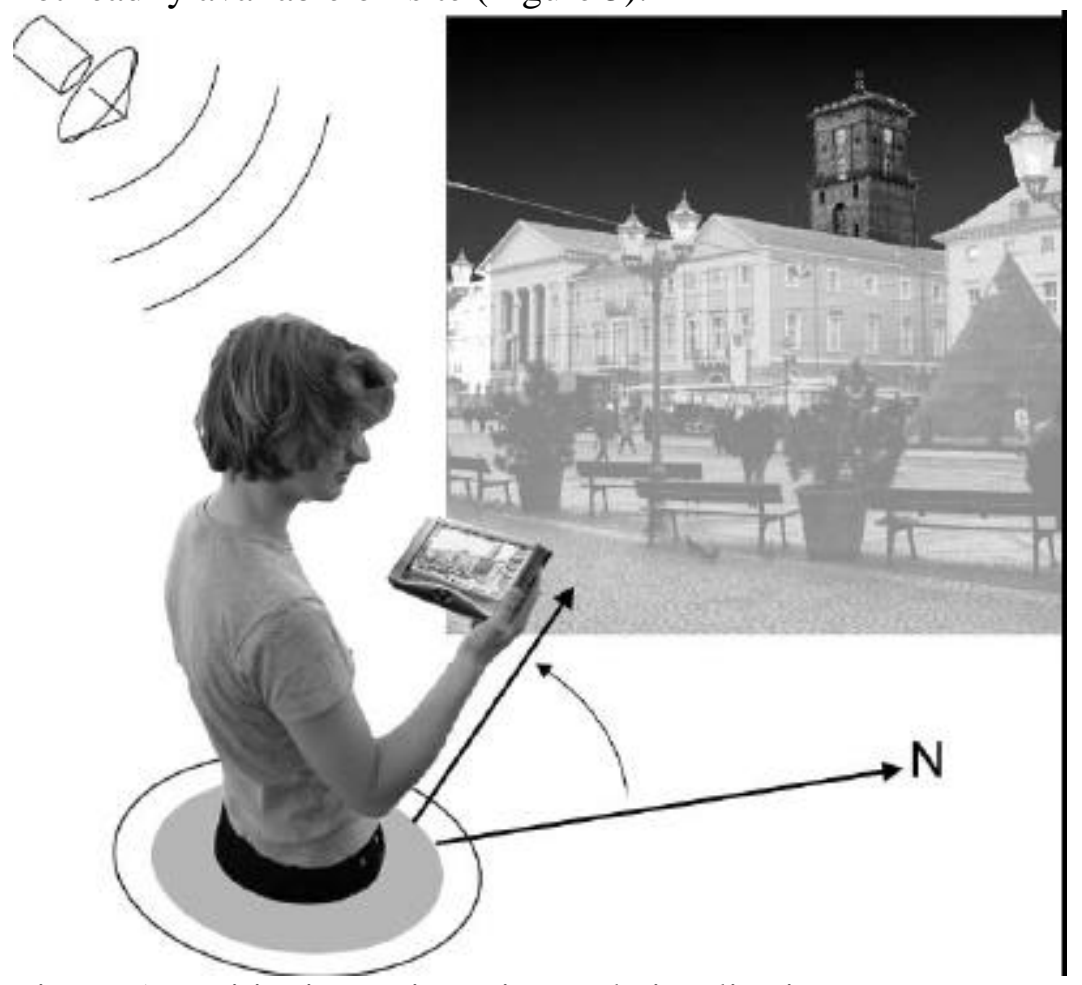

Figure 5: Positioning, orientation and visualisation.

Location, direction and time controlled transmission of context-based information whilst on location in the real environment has multiple uses for citizens, city administration, designers and planning authorities. The system could be able to make available threedimensional models of historical, present and future buildings, with linked photographic, audio, text, expert knowledge, subjective reports of time witnesses and location-based services such as entertainment facilities.

\subsection{Past, present and future}

The past aspect of the project will facilitate the creation of a collective urban memory with (edited) data entered by citizens, city administrators, designers and planners. This information will represent both the structural and cultural urban history and be accessible from within the real urban environment. However, the incorporation of user-entered data that is accessible to all users raises issues of redundancy, relevance and accuracy. The present aspect of the system will integrate existing mobile services such as Internet and telephony with mobile access to city information and service facilities such as events, gastronomy and entertainment.

The future aspect will support decision-making during urban project development by providing access to both current and historical development patterns, contextual information and culture. 


\section{POTENTIALS AND CONSTRAINTS}

The increasing spread and effectiveness of wireless data networks make the use of mobile computing devices in a city context more viable. It is foreseeable that this technology will form the basis for many new applications as transfer rates increase and costs fall. However, it is important to examine any new service in light of the real value it will add to the user.

In the past much of the research in this area has focussed only on aspects of presence (Location Based Services). The visualisation of non-visual data, such as the past and future conditions of the city and their direct integration to the real place, form the basis for a new kind of 'city access'. This opens up the data available to visitors and inhabitants. The projects described above reveal some of the potential of these possibilities and show how the ideas may be translated into action.

We conclude by summarising some of the important potentials and constraints that the projects described in this paper have revealed:

(i) The pseudo-3D model at the core of navigation in Project 1 is very small in terms of file size. Consequently, it can be transmitted quickly across wired and wireless networks, with, effectively, no time or cost overhead when compared to a $2 \mathrm{D}$ map.

(ii) The time-line feature described in Project 2 allows users better access to historic and context-specific information on urban fragments. That kind of information enhances design and planning discussions.

(iii) Partly off-line systems [21] can have some advantages over 'always on' approaches [14]. A system that is not entirely focussed on wireless networking seems advisable.

(iv) Scrolling can be difficult with small screen devices, on-site. The automatic panning feature in Project 1 helps to address this problem.

(v) Manual inputting of data and search information on a PDA on site is slow, and can be difficult. Voice recognition systems have the potential to ease these problems.

(vi) In the outdoor environment sunlight can reduce contrast and brightness of screen images and the small screen information displays have to be designed with this in mind.

(vii) An area only touched on in this paper is that of how to provide location and routing information most effectively. The $3 \mathrm{D}$ representation is useful, but others (e.g. [22]) have looked at techniques to reduce information to an optimal level, in terms of understandability and data size.

The experience and investigations undertaken with, and as a consequence of, the work in Projects 1 and 2 begin to demonstrate the potential of mobile city information systems. The seven points immediately above suggest some important issues that need to be taken into account and responded to in developing such systems further. 


\section{References}

1. Saarinen, E., The City, Its Growth Its Decay Its Future, MIT Press, Cambridge, Massachusetts, 1965.

2. Clark, H.H., Using Language, Cambridge University Press, Cambridge, 1996.

3. Healey, P., Collaborative Planning, Macmillan Press, London, 1997.

4. Hughes, Q., Seaport: The Architecture and Townscape of Liverpool, The Bluecoat Press, Liverpool, 1993.

5. Hughes, Q., Liverpool: City of Architecture, The Bluecoat Press, Liverpool, 1999.

6. Maver, T.W., The Virtual City, in: Proceedings of CAD \& Creativeness, Bialystock, 1996, 181-184.

7. Maver, T.W. and Petric, J. and Ennis, G. and Lindsay, M., Visiting The Virtual City, in: Proceedings of SIGraDi'2000 - Construindo (n)o Espacio Digital (Constructing the Digital Space), Rio de Janeiro, 2000, 135-139.

8. http://iris.abacus.strath.ac.uk/glasgow/ [April 2003].

9. http://www.ralphski.co.uk/3nge/ [April 2003].

10. Belz, C., Jung, H., Santos, L., Strack, R. and Latva-Rasku, P., Handling of Dynamic 2D/3-D Graphics in Narrow-Band Mobile Services, in: Virtual Worlds on the Internet, IEEE Computer Society, 1998, 147-156.

11. Raposo, A., Neumann, L., Magalhães, L. and Ricarte, I., Visualization in a Mobile WWW Environment, in: Proceedings of WebNet 97 - World Conference of the WWW, Internet and Intranet, 1997.

12. Coors, V. and Wiedmann, B., Using Wearable GIS in Outdoor Applications, in: Proceedings of the Symposium on Interactive Applications for Mobile Computing, IMC'98, Rostock, November 1998.

13. Rakkolainen, I. Vainio, T., Kupila, H., Majahalme, T. and Salmenpera, H., A GPSbased 3D City Info for Mobile Users, in: Proceedings of SCI 2001 Conference, Orlando, 2001. http://www.uta.fi/hyper/projektit/tred/ [April 2003].

14. Umlauft, M., Pospischil, G., Niklfeld, G. and Michlmayr, E., LoL@, a Mobile Tourist Guide for UMTS, in: Werthner, H. and Veit, E., eds., Information Technology \& Tourism, Cognizant, USA (to be published). http://lola.ftw.at/homepage/content/a40material/ [April 2003].

15. Berridge, P., Brown, A.G.P. and Knight, M.W., One City to Go: A Multi-Modal Approach to Delivering City Data, in: Proceedings of 7th International Conference on Computer Aided Architectural Design Research in Asia, Prentice Hall, Kuala Lumpur, Malaysia, 2002, 57-64.

16. Knight, M. and Brown, A.G.P., Working with Virtual Environments Through Appropriate Physical Interfaces, in: Architectural Computing: From Turing to 2000 17th Conference on Education in Computer Aided Architectural Design in Europe, University of Liverpool, 1999, 431-436.

17. http://www.liverpoolarchitecture.com/tours/ [April 2003].

18. Davies, N., Mitchell, K., Cheverst, K. and Blair, G., Developing a Context Sensitive Tourist Guide, in: Proceedings of the First Workshop on Human Computer Interaction for Mobile Devices, Glasgow, 1998.

http://www.dcs.gla.ac.uk/ johnson/papers/mobile/HCIMD1.html [April 2003]. 
19. Berridge, P. and Brown, A.G.P., A Touring Machine, in: Proceedings of [design education] 20th Conference on Education in Computer Aided Architectural Design in Europe, Warsaw, 2002, 488-493.

20. Herstad, J., Van Thanh, D. and Kristoffersen, S., Wireless Markup Language As a Framework for Interaction With Mobile Communication Devices, in: Proceedings of the First Workshop on Human Computer Interaction for Mobile Devices, Glasgow, 1998. http://www.dcs.gla.ac.uk/ johnson/papers/mobile/HCIMD1.html [April 2003].

21. Cheverst, K. Experiences of Developing and deploying a Context-aware Tourist Guide: the GUIDE project, ACM MOBICOM, 2000.

22. Agrawala, M. and Stolte, C., Rendering effective route maps: Improving usability through generalization, Proc.SIGGRAPH, 2002. 